\title{
Erzählung und Geschichte
}

\author{
Johannes Robbeck
}

Wenn die Aufgabe von Historikern und Philosophen nicht darin besteht, die Geschichte als ein Faktum mit objektiver Realität zu beschreiben, sondern einen Sinn in die Geschichte zu legen, ist genauer nach der Eigenart dieses Sinns zu fragen. Dazu erinnere ich an die doppelte Bedeutung des Begriffs der Geschichte. Bekanntlich bezeichnet Geschichte sowohl die Darstellung in der Historiographie als auch den Sachverhalt zeitlich aufeinander folgender Ereignisse. ${ }^{1}$ Diesen beiden Seiten der Geschichte kann ein je verschiedener Sinn zugeordnet werden.

Einerseits bedeutet Geschichte die Darstellung, so wie man eine Geschichte oder Geschichten erzählt. Dem entspricht das aus dem Griechischen und Lateinischen stammende Wort Historie (bistoria), mit dem das über ein Gebiet versammelte Wissen gemeint ist. Hier geht es um die Methode der Forschung, um die Interpretation historischer Ereignisse und um ihre entsprechende Präsentation. Wenn der Historiker die Geschichte auf diese Weise deutet, verleiht er der Geschichte einen bestimmten Sinn. Diesen Sinn nenne ich Deutungssinn.

Andererseits verweist das Wort Geschichte auf den Sachverhalt, d. h. auf den Zusammenhang von Ereignissen, die in der Vergangenheit passiert sind (res gestae). Geschichte ist hier geschehene Geschichte, wie der etymologische Befund verdeutlicht. Damit ist der historische Prozess gemeint, der zum Gegenstand einer Darstellung wird. Fragt man nach dem Inhalt, lautet eine erste allgemeine Antwort: Geschichte besteht aus individuellen und kollektiven Handlungen der Menschen in der zeitlichen Folge. Denn jedes historische Ereignis setzt bestimmte Handlungen voraus, so wie diese selbst geschichtlich verfasst sind. Diesen Sinn bezeichne ich als Handlungssinn. ${ }^{2}$

Sofern die Darstellung der Geschichte im Vordergrund steht, ist vor allem der Deutungssinn wichtig, wie in neueren Diskurs- und Erzähltheorien beobachtet werden kann. Wenn dort ausschließlich der Deutungssinn interessiert, wird der Handlungssinn programmatisch ausgeblendet. Analog dazu ist in entsprechenden Geschichtsphilosophien der Deutungssinn das beherrschende Thema. Damit entfällt der Referent der Erzählung, was die Unterscheidung zwischen realer Geschichte und Fiktion erschwert. Für das Verhältnis von faktualem und fiktionalem Erzählen folgt daraus, dass sich die Grenze zwischen diesen beiden Erzähl-

1 Auf diese Eigenart des modernen Geschichtsbegriffs hat schon Hegel aufmerksam gemacht (Hegel 1969: XII 83).

2 Herbert Schnädelbach, dem ich hier teilweise folge, unterscheidet zwischen einem Handlungssinn und einem Mitteilungssinn (Schnädelbach 2003: 336). - Man kann auch den Handlungssinn als einen Deutungssinn bezeichnen, weil ja der Handelnde sich dabei selbst deutet. Um aber die Perspektive des Handelnden zu betonen und sie von der des Historikers zu unterscheiden, benutze ich hier den Terminus ,Handlungssinn“. 
typen so weit verschiebt, dass der Eindruck entsteht, als ob jede Erzählung, insbesondere die historische Erzählung, letztlich bloß fiktional wäre. Im Gegenzug stelle ich mir die Aufgabe, den Handlungssinn wieder stärker zur Geltung zu bringen und das faktuale gegenüber dem fiktionalen Erzählen aufzuwerten.

Natürlich wäre es zu einfach, den Deutungssinn durch den Handlungssinn zu ersetzen. Vielmehr kommt es darauf an, beide Seiten miteinander zu vermitteln. Daher möchte ich den Zusammenhang zwischen Handlungssinn und Deutungssinn untersuchen, wobei ich auf eine Wechselbeziehung ziele. Ich werde versuchen, zu zeigen, dass nicht nur der Handlungssinn im Medium der narrativen Deutung entsteht, sondern dass vor allem der Deutungssinn das Konzept einer sinnhaften Handlung voraussetzt. Die Vermittlung soll im Nachweis einer engen Korrelation bestehen, indem Handlungssinn und Deutungssinn aufeinander verweisen. Sinnstiftung heißt zunächst, dass der Historiker die in der Vergangenheit erfolgten Handlungen deutet. Aber diese Deutung ist nur möglich, wenn er zugleich über bestimmte Vorstellungen menschlichen Handelns verfügt. Das gilt auch für die Theorie und Philosophie der Geschichte, in der nicht nur das alltägliche Verständnis von Handlung, sondern Handlungsbegriffe, Handlungstypen und Handlungstheorien vorausgesetzt werden. Meine These ist, dass die Handlung kein bloßes Faktum der Geschichtsschreibung ist, sondern bereits einen eigenen historischen Sinn enthält, der in die Historiographie und Geschichtsphilosophie transformiert wird.

Um diese These zu stützen, versuche ich zunächst eine handlungstheoretische Rekonstruktion der unterschiedlichen Methodologien in der Geschichtswissenschaft und Philosophie der Geschichte. Ich werde zeigen, auf welche Weise die sich wandelnden Auffassungen über menschliches Handeln die aufeinander folgenden Methodenreflexionen beeinflussen. Im Anschluss an den Historismus des 19. und die Erzähltheorien des 20. Jahrhunderts soll außerdem demonstriert werden, wie Handlungssinn und Deutungssinn mit unterschiedlicher Nähe und Ferne wechselseitig oszillieren. Sodann werde ich mit Hilfe der Theorie der dreifachen Mimesis von Paul Ricœur die systematische Vermittlung weiter entwickeln.

\section{Handlungssinn und Deutungssinn}

Es gibt gute Gründe, die moderne Theorie der Geschichtserzählung in die hermeneutische Tradition des Historismus zu stellen. Besteht doch eine Gemeinsamkeit in der Grundauffassung, dass die historischen Ereignisse nicht einfach gegeben sind, sondern vom Historiker interpretiert und deutend dargestellt werden. Schon Johann Gustav Droysen hatte in seiner Historik zwischen ,Forschung' und ,Darstellung' unterschieden und die Funktion der historischen Erzählung erkannt (Lorenz 1997: 127 ff.; Droysen 1977: 130 ff.). In diesem Sinne ist die Erzählung eine interpretierende Darstellung eines historischen Ganzen. Von dort hat sich eine überraschend enge Verbindung zu bestimmten Theorien historischer Erklärung in 
der analytischen Philosophie herausgestellt, die eine Übereinstimmung von hermeneutischem Verstehen und intentionaler Erklärung aufdeckten. Weiter spannt sich ein Bogen zur analytischen Philosophie der Geschichte, die ja nicht das Erzählen gegen das Erklären ausspielt, sondern ihren eigenen Typ des narrativen Erklärens beansprucht. Aus diesen Gründen liegt es nahe, nicht nur an die Hermeneutik, sondern auch an die analytische Erzähltheorie anzuknüpfen.

Bei näherer Betrachtung zeigt sich, dass diese Brücke für uns nicht gangbar ist. Denn aus der Perspektive der Handlung tut sich an dieser Stelle ein tiefer Graben auf. Während sich der alte Gegensatz zwischen Erklären und Verstehen durch das intentionale Erklärungsmodell überbrücken lässt (Droysen 1977: 164; Wright 1974: 90; ausführlich hierzu Haussmann 1991: 189 ff.; vgl. Riedel 1978: 165 ff.; Lorenz 1997: 107 f.), ist der neue Gegensatz zwischen intentionaler und narrativer Erklärung unüberwindbar. Denn die Erzählung erklärt eine Handlung nicht mehr mit dem Verweis auf die Motive oder Ziele des Handelnden, sondern vom Ergebnis her. Damit wird die Intention einer Handlung ausdrücklich eliminiert. Diese radikale Wende soll kurz mit Arthur C. Danto wie auch Hayden White und etwas ausführlicher anhand von Paul Ricœur entfaltet werden. Die Pointe meiner Untersuchung besteht jedoch darin, dass sich bei Ricœur, obwohl er zu den bekanntesten Narratologen gehört, zugleich die gegenläufige Absicht herauslesen lässt, die Eigenständigkeit der Handlung gegenüber der Erzählung zu retten.

In seiner Analytischen Philosophie der Geschichte, die sich sowohl gegen die traditionelle Geschichtsphilosophie als auch gegen das naturwissenschaftliche Erklärungsmodell von Carl G. Hempel richtet (Hempel 1959), will Danto weder Aussagen über die Geschichte „im Ganzen“ wagen, noch Gesetzesaussagen analysieren (Danto 1974: 11 f.; vgl. Ankersmit 2009: 132). Vielmehr behandelt er „erzählende Sätze“, denen er gleichwohl eine erklärende Funktion zuschreibt. Doch verbindet Danto die historischen Ereignisse nicht durch einen Kausalnexus, sondern verleiht den historischen Ereignissen eine Bedeutung, die er als "historische Signifikanz“ bezeichnet, indem er ihre Stelle innerhalb der Reihenfolge der erzählten Ereignisse markiert (Danto 1974: 232 ff., 269, 374; vgl. Schiffer 1980: 23 ff.). Wenn eine Erzählung dem Schema „Anfang, Mitte, Ende“ folgt, ist für die Deutung eines mittleren Ereignisses entscheidend, dass es nur vom Ende der Erzählung her verständlich wird. Wenn ein Historiker beispielsweise schreibt: „Der Dreißigjährige Krieg begann im Jahr 1618“, gewinnt dieser Satz erst aus der rückblickenden Betrachtung seinen Sinn. Die Beteiligten konnten sich über die Tragweite und Bedeutung ihres Handelns nicht im Klaren sein; der Begriff „Dreißigjähriger Krieg“ erschließt sich erst aus der Kenntnis der abgeschlossenen Entwicklung. „Allgemein gesprochen: was wir als Anfang einer Erzählung auswählen, ist bestimmt durch das Ende“ (Danto 1974: 394). Das führt ständig zur „rückwirkenden Neugliederung“ oder gar zur „Re-Strukturierung der gesamten Geschichte“ (270).

In unserem Zusammenhang ist nun interessant, welche Rolle dabei der Handlung zugeschrieben wird. Danto räumt ein, dass die Erzählung eine bestimmte 
Vorstellung von Handlung voraussetzt. Wenn zum Beispiel erzählt wird, dass jemand eine „Rose pflanzt“, setzt diese Erzählung die Kenntnis der Handlung „Rosenpflanzen“" voraus (258). Aber die Absicht einer solchen Handlung blendet Danto aus, weil er die Aktionsreihe ausschließlich aus der Erzählperspektive deutet. Entscheidend für die Geschichtsdeutung soll nicht das anfängliche Motiv des Handelnden sein, sondern das Resultat, das bei längeren Handlungsketten nur der Historiker kennt. Danto will nicht den Sinn „in der Geschichte“, sondern den Sinn „der Geschichte" untersuchen, d. h. denjenigen Sinn, der sich aus dem Blick des Historikers auf die Geschichte ergibt.

Allerdings ist die Ausblendung der Intention nur mit bestimmten Zusatzannahmen plausibel, die nicht explizit werden. Wenn das Motiv des Handelnden mit dem Ergebnis der Handlung übereinstimmt, lässt sich die narrative Erklärung auch als teleologische oder intentionale Erklärung im Sinne eines hermeneutischen Verstehens lesen. ${ }^{3}$ Das Ende ist dann die Realisierung einer anfänglichen Zielsetzung oder der finale Ausdruck einer ursprünglichen Intention. Nur wenn das Resultat von der Intention abweicht, schreibt der Erzähler der Handlung einen anderen Sinn zu, der sich aus der Kenntnis der späteren Wirkung ergibt. In diesem Fall, der in der Geschichte die Regel ist, erhält die Erzählperspektive ihre volle Berechtigung. Die Stärke der Narratologie besteht daher in der Berücksichtigung der historischen Kontingenz. Die narrative Erklärung entspricht hier einer funktionalen Erklärung, die zeigt, mit Hilfe welcher Faktoren ein späterer $\mathrm{Zu}$ stand aus einem früheren entstanden ist.

Doch ist nicht einzusehen, warum die Handlungsmotive überhaupt keine Rolle mehr in der Geschichtserzählung spielen sollen. Denn die Feststellung kontingenter Verläufe setzt die Analyse der Abweichung zwischen Motiv und Resultat voraus. Allein die Feststellung, ein Tyrannenmord ist gescheitert (Wright 1974: 93), unterstellt eine entsprechende Absicht. Bleibt hingegen der Handlungssinn ausgeklammert, beschränkt sich die erzählende Geschichtsschreibung auf den Deutungssinn.

Auch Hayden White betrachtet die Erzählung als konstitutiv für die historische Erkenntnis. In seiner Metahistory geht er noch einen Schritt über Danto hinaus, indem er die temporale Struktur des Vor- und Nacheinanders durch einen eigenen Inhalt, ein Thema oder eine Fabel ergänzt.

Die erste naive Form der historischen Darstellung bilden die mittelalterlichen Annalen (White 1991: 19 ff.; 1990: 17 ff.; vgl. Müller-Funk 2002: 130 ff.). Sie verfügen nur über das Schema der aufeinander folgenden Jahre, denen jeweils bestimmte Ereignisse zugeordnet werden. Eine höhere Form der Geschichtsschreibung ist die Chronik, die bereits einen größeren Umfang und einen gemeinsamen Themenbereich enthält, wie die Chronik einer Stadt oder Region. Doch ist die Chronik noch der zeitlichen Folge verhaftet, so dass ihr die Ge-

3 Ricœur wird später von „narrativem Verstehen“ sprechen (Ricœur 1988-1991: I 92). 
schlossenheit einer Erzählung fehlt. Von diesen Vorformen hebt sich nach White die eigentliche Historie ab, deren Geschichtserzählung eine sinnvolle Ganzheit mit einer geschlossenen Zeitstruktur von Anfang, Mitte und Schluss aufweist. Das verdankt sich einer „Fabel“, die in die Erzählung eingefügt wird und ihr einen moralischen Sinn verleiht. Wie bei Danto erfüllt die Fabelkomposition auch eine Erklärungsfunktion, indem begründet wird, warum ein bestimmtes historisches Ereignis eingetreten ist.

Indem White die Erzählung für konstitutiv erklärt, trennt er die Historiographie von der historischen Forschung ab und behandelt allein die „Bedeutung der Form“ der Darstellung (White 1990: 12 ff.). In dieser Sichtweise bilden die historischen Fakten lediglich den „Grundstoff“, der nach literarischen Mustern kombiniert wird. White zufolge unterscheiden sich Erzählungen von Historikern, Geschichtsphilosophen und Romanschreibern nur noch graduell.

Das Problem dieser Konzeption liegt im Verhältnis von Historiographie und literarischer Fiktion. Radikalisiert man die Diskurstheorie, löst sich die Bedeutung eines Textes von seiner Referenz auf eine Wirklichkeit. Die Geschichtserzählung erscheint wie ein autonomes Gebilde, das durch keine realen Kontexte eingeschränkt wird. Gegen White ist einzuwenden, dass mit der ausschließlichen Konzentration auf Fragen der literarischen Form der Verweis eines historischen Textes auf historische Ereignisse außerhalb von Texten verdrängt wird. So ist es nicht verwunderlich, dass White in einer späteren Verteidigung seiner Metahistory die Behauptung revidiert, historische und fiktionale Darstellung seien grundsätzlich ununterscheidbar (White 1986: 101 ff.; vgl. Rohbeck 2000: 60 f.).

Wenn die historischen Fakten derart relativiert werden, spielen auch die wirklichen Handlungen und deren Motive in der Geschichtserzählung keine Rolle mehr. Es wird zwar von bestimmten Handlungen erzählt, aber deren Sinn soll sich allein aus der literarischen Fiktion ergeben. Damit reduziert sich der Handlungssinn auf den Deutungssinn der Erzählung. Doch wenn man weiter fragt, woraus diese Art Deutung ihren Sinn schöpft, stellt sich heraus, dass die literarischen Formen der Geschichtserzählung in Wahrheit auf bestimmte Grundtypen menschlichen Handelns rekurrieren. Die Formen Romanze, Komödie, Tragödie und Satire enthalten je spezifische Handlungsmuster (White 1991: 21 ff.; zur Romanze 179 ff., zur Komödie 214 ff., zur Tragödie 251 ff., zur Satire 302 ff.), die auf die erzählte Geschichte übertragen werden. So enthält die Tragödie gewissermaßen die Urform eines moralischen Konflikts, wie Hegel am Beispiel der $A n$ tigone von Sophokles zeigt (Hegel 1969: III 383 ff., 417 ff.; 1969: VII §§ 118, 166). Die dramatische Dichtung liefert das elementare Handlungsmodell. Daraus folgt für die literaturwissenschaftliche Konstruktion, dass es ein Vorverständnis menschlicher Handlung gibt, das aus der praktischen Erfahrung stammt und über das Medium der Literatur in die Geschichtserzählung eingeht.

Einen weiteren Schritt in der Erzähltheorie der Geschichte geht Paul Ricœur. White und Ricœur haben ihre Theorien wechselseitig rezipiert und kommentiert. 
Wie sich Ricœur von White inspirieren ließ, so würdigte White wiederum Ricœurs Untersuchungen zum Verhältnis von Erzählung und Zeitlichkeit. Er glaubt sich mit ihm darin einig, dass die geschichtliche Zeit nur narrativ erschlossen werden könne. Wichtig sei nicht mehr, so White, ob Historiker realen Ereignisfolgen, die ebenso gut auch nicht narrativ darstellbar wären, eine „narrative Form " auferlegen, sondern ob historische Ereignisse dieselbe Struktur besitzen wie narrative Diskurse (White 1990: 177 f.; Ricœur 2004: 391 ff.; vgl. Stückrath/Zbinden 1997). Mit dieser Analogie behauptet White eine Einigkeit, mit der er Ricœur für seine Position zu vereinnahmen versucht. Es ist hingegen meine Absicht, so weit wie möglich den Unterschied zwischen White und Ricœur herauszuarbeiten, ja sogar Ricœur, gegen den Strich` zu lesen.

Mit seinem Werk Zeit und Erzäblung, besonders dem ersten Band Zeit und historische Erzäblung, rückt Ricœur das Thema Erzählung ins Zentrum. Damit kritisiert er Geschichtstheorien der analytischen Philosophie, in denen etwa Hempel versucht hatte, historische Ereignisse nach dem Vorbild der Naturwissenschaften zu erklären. Ausdrücklich knüpft er an Danto an, dessen narrativistischen Ansatz er mit Hilfe der hermeneutischen Methode erweitert. Mit seiner eigenen Theorie historischer Erzählung beabsichtigt Ricœur zweierlei: Erstens will er zeigen, dass sich Erklären und Erzählen keineswegs ausschließen, und zweitens, dass sich Zeiterfahrung und Erzählung wechselseitig bedingen.

Ricœur erkennt in der analytischen Theorie der Erzählung eine wichtige Grundlage für die gesuchte Synthese von Erklären und Erzählen, die er „narrativistische Argumente" nennt (Ricœur 1988-91: I 214, 268; vgl. 2004: 366 ff.). Seiner Auffassung nach können Erzählungen etwas erklären, wie umgekehrt auch Erklärungen eine narrative Struktur besitzen. Indem eine Erzählung das Vorher und Nachher, die Folge einer Handlung aus einer vorhergehenden Handlung, die Motive und Umstände der Handlungen zu einer sinnvollen Einheit komponiert, werden historische Ereignisse nicht nur beschrieben, sondern auch „erklärt". Wie bei Danto erschließt sich für Ricœur die Bedeutung eines Ereignisses erst vom „Endpunkt“, der zugleich den „Abschluss“ einer Geschichte „in finaler Hinsicht" bildet (Ricœur 1988-91: I 108 f.; 2004: 366). Gleichwohl geht ihm dieser Ansatz nicht weit genug, weil er sich auf einzelne Sätze beschränke und die Fabel von Geschichten unberücksichtigt lasse.

Im Unterschied zu Danto legt Ricœur seiner Erzähltheorie die Komposition einer Fabel zugrunde. Wie schon Droysen bezeichnet er diese Art Fiktion als „mimesis“ (Ricœur 1988-91: I 104), um den literarisch-künstlerischen Aspekt zu betonen. Zur Deutung gehört daher nicht nur die Reihenfolge der Ereignisse, die aus rückblickender Perspektive betrachtet werden, es bedarf vielmehr einer bestimmten inhaltlichen Figuration. Diesen Aspekt der „Fabelkomposition“ entlehnt Ricœur von White (Ricœur 1988-91: 242 ff.). Die originelle Leistung von Ricœur besteht darin, dass er die Fabelkomposition in einen größeren Zusammenhang stellt, den er mit Hilfe der Hermeneutik anreichert (Müller-Funk 2002: 
69, 70 ff.). Indem er die bloß immanenten Konzeptionen der Narratologie überwindet, erklärt er die Erzählung zur geschichtlichen Praxis.

Damit eine Fabel überhaupt von einem Leser oder Hörer verstanden werden kann, so argumentiert er, bedarf es sowohl eines bestimmten Vorverständnisses als auch einer aktiven Verarbeitung und Rückführung auf die eigene Zeit- und Lebenserfahrung. Die in der Mitte angesiedelte Konfiguration der Fabel heißt „mimesis II“, deren Voraussetzungen und Konsequenzen nach beiden Seiten hin untersucht werden.

Die vorausgesetzte Präfiguration der „mimesis I“ besteht in der Welt der Handelnden. Der Leser einer Erzählung muss mit den Strukturmerkmalen einer Handlung aus praktischer Erfahrung vertraut sein (Ricœur 1988-91: I 90 f.). Die abschließende Refiguration der „mimesis III“ folgt dem hermeneutischen Grundsatz, dass die Bedeutung einer Erzählung nicht nur vom schreibenden Autor, sondern auch vom verstehenden Leser konstruiert wird. Hier geht es um die „Schnittstelle“ zwischen der Welt des Textes und der Welt des Lesers oder Hörers. Die Erzählung erlangt erst ihren vollen Sinn, wenn sie in die Erfahrung des Lesers überführt wird (114).

Ricœur ist sich der Gefahr bewusst, damit die Grenzen zwischen literarischer Fiktion und historischer Wahrheit zu verwischen. Doch an dieser prekären Stelle folgt er nicht mehr White, was in Zeit und Erzäblung daran zu erkennen ist, dass er den Referenten wirklicher Ereignisse nicht aufzugeben bereit ist, indem er die Geschichte als eine „Quasi-Fabel“ bezeichnet $(298,344)$.

Im späteren Werk Gedächtnis, Geschichte, Vergessen verstärkt sich diese Distanzierung von White zugunsten des Anspruchs auf historische Wahrheit. Ähnlich wie Droysen lässt Ricœur die historiographische Tätigkeit mit der Erhebung von Fakten beginnen, fühlt sich doch der Historiker verpflichtet, dem Leser vom „Realen“ zu berichten (Ricœur 2004: 207 ff., 427). Ricœur unterscheidet deutlich zwischen einer „Repräsentation als Operation, die ein Moment des GeschichteErzählens ist“ und einer „Repräsentation als Objekt, die ein Moment des Geschichte-Machens ist" (Ricœur 1997: 442; 2004: 354). Insbesondere das historische Gedächtnis erhebt einen Wahrheitsanspruch, der sich auf die Vergangenheit als ihren Referenten bezieht (Ricœur 1998: 25; 2004: 25). Schließlich hängt dieser „Realismus des Zeugnisses“ mit der ethischen Verpflichtung gegenüber den Opfern der Geschichte zusammen; er besteht in der Anerkennung von Schuld in der Geschichte und berührt das Problem des Vergessens, der Vergebung und des Verzeihens (Ricœur 1998: 131 ff.; 2004: 397, 432). In diesem Kontext berühren sich Geschichtsphilosophie und Ethik (Rohbeck 2010: 106).

Doch im epistemologischen Zusammenhang interessieren mehr die Konsequenzen des Realismus für die Beziehung der Geschichtserzählung zur menschlichen Handlung. Wie erwähnt, strebt Ricœur eine Vermittlung zwischen „Zeit und Erzählung“" an, die er als Korrelation auffasst. Doch bereits auf der ersten Stufe der Mimesis wird klar, dass Zeit dort zunächst die "Zeit der Handlung“ 
(Ricœur 1988-91: I 130) bezeichnet, d. h. diejenige Zeit, die durch die Handlung strukturiert wird. In der Vermittlung von Zeit und Erzählung geht es also um die Vermittlung von Handlung und Erzählung, bzw. um die Vermittlung von Handlungszeit und Erzählzeit.

$\mathrm{Da}$ in der Mimesis das Vorverständnis von Handlung wesentlich ist, gilt die Fabel nicht etwa als Konstruktion von Handlung, sondern ist „Handlungsnachahmung" (Ricœur 1988-91: I 90-93). Daher wird beim Erzähler die Fähigkeit vorausgesetzt, die Strukturmerkmale einer Handlung überhaupt zu erkennen. Aus diesem Grund stellt sich Ricœur zunächst die Aufgabe, einen Begriff von Handlung zu entfalten, der Subjekt, Zweck und Mittel sowie die zeitliche Dimension umfasst (Ricœur 1988-91: I 133 f.; 2004: 542 ff.). Diese Komponenten machen das „praktische Verstehen“ aus. Davon unterscheidet er das „narrative Verstehen“, das einer anderen Regel folgt und, wie bei Danto, die Kette der Handlungen umgekehrt vom Ende zum Anfang der Geschichte liest. Doch im Unterschied zu Danto betont Ricœur, dass die Kompositionsregel der Erzählung die fundamentale Ordnung der Handlungszeit keineswegs aufhebt. Daraus folgt die weitreichende These, dass die Handlung eine „pränarrative Struktur" besitzt, dass sie im Kontext der praktischen Verflechtung in der Lebenswelt eine „Vorform der Erzählung" darstellt (Ricœur 1988-91: I 98 f., 118).

Aus der Rehabilitierung der Handlung gegenüber der Erzählung folgt, dass der Historiker nicht nur eine „wahre“ Geschichte erzählen, sondern bestimmte „Handlungsträger" in der Geschichte zugrunde legen soll. Dazu gehört ausdrücklich, ihre Motive zu berücksichtigen und sie als „kompetente Akteure“ ernst zu nehmen (Ricœur 2004: 344 f., 354, 592). Ricœur zufolge hat der Geschichtsschreiber „sich an den Augenblick der Handlung zurückzuversetzen und sich zum Zeitgenossen des Handelnden zu machen“ (Ricœur 1997: 446 f.). ${ }^{4}$ Diese Forderung hält er mit der Kontingenz in der Geschichte für vereinbar. Während Danto aus Gründen der historischen Kontingenz die intentionale durch die narrative Erklärung ersetzt, legt Ricœur besonderen Wert darauf, dass trotz der Einsicht in die Kontingenz und trotz des Paradigmenwechsels zur Narration der intentionalen Erklärung ihr angestammtes Recht erhalten bleibt.

Damit gewinnt die Handlung gegenüber der Erzählung eine größere Relevanz. Nur unter der Voraussetzung einer relativen Eigenständigkeit des Verständnisses von Handlung kann überhaupt von einer Vermittlung von Zeit und Erzählung bzw. Handlungszeit und Erzählzeit die Rede sein. An diesen Ansatz möchte ich mich in meinem Versuch einer Vermittlung von Handlungssinn und Deutungssinn anschließen.

4 Ricœur zitiert hier zustimmend Raymond Aron (1948); zur Kontingenz vgl. auch Ricœur (1988-1991: I 108). 


\section{Die dreifache Vermittlung}

Das Verhältnis von Zeit und Erzählung bei Ricœur konnte so rekonstruiert werden, dass die Zeitstruktur nicht allein von der Erzählung bestimmt wird, sondern auch und primär von der menschlichen Handlung. Interessant war, dabei zu beobachten, wie Ricœur gerade in seiner Narratologie den Aspekt der Handlung wieder angemahnt hat. Und in seiner Theorie des Gedächtnisses hat er das Insistieren auf die Handlung mit einem Realismus in der Geschichtswissenschaft verbunden. Ich folge dieser Theorie, indem ich die Figur der „dreifachen Mimesis“ auf die Vermittlung von Handlungssinn und Deutungssinn anwende (Ricœur 1988-91: I 87 ff.).

Auf der ersten Stufe (mimesis I) liegt ein bestimmtes Vorverständnis von Handlung vor. Es gründet sich auf ein ,praktisches Verstehen“, das von der Alltagserfahrung geprägt wird, und unterscheidet sich deutlich vom „narrativen Verstehen", das aus der Erzählperspektive resultiert. Auf der zweiten Stufe (mimesis II) wird dieser Handlungssinn in eine Geschichtserzäblung transformiert. Der Sinn der dort handelnden Menschen wird nicht aus dem Nichts kreiert, vielmehr wird ein Handlungssinn vorausgesetzt und narrativ überformt. Der Erzähler entfaltet dessen historische Potenziale, indem er eine im Handlungssinn bereits angelegte virtuelle Geschichte realisiert (Ricœur 1988-91: I 118; Bubner 1984: 23). Durch diese Art der Interpretation wird der Handlungssinn in einen Deutungssinn überführt. Auf der dritten Stufe (mimesis III) erzeugt der Leser einen je eigenen Sinn, indem er den Deutungssinn wieder auf die eigene Lebenserfahrung und damit auf den vorgängigen Handlungssinn zurückführt.

Diese dreifache Vermittlung hat zum Ziel, dem Handlungssinn gegenüber dem Deutungssinn mehr Gewicht zu verleihen. Allein die Unterscheidung zwischen Handlungssinn und Deutungssinn signalisiert diese Absicht. Gegenüber einer rigorosen Diskurstheorie oder einem extremen Konstruktivismus stellt der Begriff des Handlungssinns schon eine Provokation dar, weil er der Handlung in relativer Unabhängigkeit von der Historiographie einen eigenen Sinn zuschreibt. Dieser spezifische Sinn der Handlung besteht auf der Seite der Handlung in den Motiven und Intentionen der Handelnden und in den jeweiligen Deutungsmustern, von denen die Handlung geleitet wird. Der Handlungssinn besteht auf der Seite der Deutung im Vorverständnis von Handlungen sowie in Deutungsmustern, die sich aus praktischen Erfahrungen herleiten lassen.

In der Geschichte des historischen Denkens zeigt sich dabei eine eigenartige Verschiebung innerhalb des Verhältnisses von Handlungssinn und Deutungssinn. Der Akzent verschiebt sich wechselnd nach beiden Extremen, je nachdem, was unter einer Handlung verstanden wird. Sofern die Motive und Absichten der historisch Handelnden wichtig genommen werden, liegt der Akzent auf dem Handlungssinn. Wird hingegen angenommen, dass die Akteure die Wirkungen ihrer Handlungen nicht überschauen und vorhersehen, liegt die Deutungskompetenz beim Historiker bzw. Erzähler. 
In diesem Zusammenhang kann man von einer unterschiedlichen Nähe und Ferne zwischen Handlungssinn und Deutungssinn sprechen. Wenn unterstellt wird, dass der Zweck bzw. das Ziel einer historischen Handlung realisiert wird, also am ,Ende' herauskommt, was ursprünglich intendiert wurde, decken sich Handlungssinn und Deutungssinn. Die Deutung läuft darauf hinaus, die Intention des historischen Akteurs zu bestätigen. Wenn hingegen festgestellt wird, dass etwas anderes folgt, als vom Handelnden beabsichtigt wurde, dann widerspricht der Geschichtsschreiber dem Selbstverständnis des Handelnden und macht seinen eigenen Deutungssinn geltend. In diesem Fall driften Handlungssinn und Deutungssinn auseinander.

Diese Art Vermittlung von Handlungssinn und Deutungssinn möchte ich nun noch weiter präzisieren. Ich beschreibe sie genauer auf drei Ebenen: auf der Ebene der Historiographie, der Theorie der Geschichte und der pragmatischen Geschichtsphilosophie.

\section{Historiographie}

Die erste Ebene betrifft die Arbeit des Historikers, der konkrete Handlungen von bestimmten Akteuren in der Geschichte deutet; es ist die Ebene der Geschichtswissenschaft, d. h. der historischen Forschung, Darstellung oder Erzählung. Mimesis I und II heißt in diesem Fall:

Zweifellos muss eine historische Handlung vom Historiker gedeutet werden. Der Handlungssinn erschließt sich nur im Modus des Deutungssinns. Und diese Deutung unterscheidet sich von den Motiven und Sinngebungen der Handelnden, weil der Interpret mit zunehmendem zeitlichem Abstand mehr weiß als der historische Akteur. Aus hermeneutischer Perspektive lässt der Historiker die Erfahrungen seiner Gegenwart in die Deutung einfließen; aus der Erzählperspektive ordnet er die Handlungen vom Standpunkt des ,Endes' ein und verleiht ihnen dadurch ihren Deutungssinn. Was Danto am Beispiel des langen Kriegs im 17. Jahrhundert erläutert, gilt auch für die jüngst von Historikern vertretene Sicht, der Erste und Zweite Weltkrieg bildeten zusammen den Dreißigjährigen Krieg des 20. Jahrhunderts. Dabei werfen die Erfahrungen sowohl dieser Katastrophe als auch des geeinten Europas ein völlig anderes Licht auf diese Kriege, als das je zuvor möglich gewesen wäre.

Aber der Deutungssinn des Historikers ist nicht willkürlich, er erschöpft sich nicht in Diskursivität, sondern hat in der historischen Handlung ihren Referenten. In der Geschichtswissenschaft dürfte dieser Realismus eine Selbstverständlichkeit sein. Demnach ist die Geschichte eben kein bloßes „Konstrukt“, sondern setzt Ereignisse in der Zeit voraus, auf die sich der historische Diskurs bezieht. Für die „Angemessenheit“ der Deutung gegenüber der geschehenen Geschichte lassen sich durchaus Kriterien formulieren, wie etwa die Forderung, dass historische Aussagen den Quellen nicht widersprechen dürfen (Kocka 1989: 8 ff.; 
Schnädelbach 1987: 13 f.; 2003: 347; Dux 1989: 38 f.; Lorenz 1997: 17 ff.). Daher gibt es weder die reine Faktizität noch den reinen Diskurs, wohl aber Deutungen von Handlungen, die als historische Fakten anzuerkennen sind.

Dieser Handlungssinn der ,real ${ }^{6}$ in einer historischen Situation Handelnden besteht zunächst in der Struktur einer Handlung, die das Subjekt, das Ziel und Motiv, die Umstände, die Interaktion und den Ausgang menschlichen Handelns ausmacht, dem symbolischen Charakter, der im kulturellen Kontext der Beschreibung liegt, und den zeitlichen Dimensionen. Indem die Handlungen eine Verbindung von Vergangenheit, Gegenwart und Zukunft herstellen, erzeugen sie bestimmte Zeitstrukturen, die durch erinnernde Reflexion ihren spezifisch geschichtlichen Charakter erhalten.

Die Aufgabe des Historikers sieht Ricœur darin, die „praktische Kompetenz“ der Akteure anzuerkennen - eine Aufgabe, die durch die Einsicht in die Kontingenz der Geschichte keineswegs obsolet geworden ist. Wie sich bei Danto zeigt, setzt die Feststellung kontingenter Verläufe die Analyse der Abweichung zwischen Motiv und Resultat voraus. Gerade aus der Erforschung von Zielen, die nicht verwirklicht werden konnten oder die zu anderen Ergebnissen führten, lassen sich wichtige Aufschlüsse gewinnen. Emphatisch gesprochen, verbindet sich damit eine Wiederkehr des historischen Subjekts (Müller-Funk 2002: 70). Das hat nicht nur eine epistemologische Seite, sondern, wie besonders Ricœur zeigt, auch eine ethische Bedeutung. Die Erzählung hat die Funktion, die Welt „bewohnbar“ (Ricœur 1988-91: I 9) zu machen. Jede Erzählung bezieht sich darauf, dass wir handelnd in der Welt sind. Die Erzählung selbst wird zur geschichtlichen Praxis.

Bisher wurde versucht, zu zeigen, dass der Handlungssinn Potenziale enthält, die in Geschichtserzählungen realisiert werden. Ein solcher prä-narrativer Handlungssinn wird dort nicht erst geschaffen, sondern in einen narrativen Kontext transformiert und dadurch modifiziert. ${ }^{5}$ Dieser Sinn ist die Bedingung der Möglichkeit historischer Darstellungen, mithin das Transzendental jeder weiter gehenden Reflexion über die Geschichte. ${ }^{6}$

5 Wie es präreflexive oder präpositionale Vorstellungen gibt, spreche ich von einem pränarrativen Handlungssinn, indem ich mich an Ricœurs Begriff einer „pränarrativen Struktur der Erfahrung“ anlehne und die Begriffe „präfigurierte Zeit“ oder „quasi-narrative Bedeutung" modifiziere (Ricœur 1988-91: I 89, 118; 2004: 9 f., 557, 594).

6 Damit grenze ich mich von anderen Entwürfen einer transzendentalen Geschichtsphilosophie ab: Bei Kant stellt die „Naturabsicht“ eine „regulative Idee“ der Geschichtsbetrachtung dar (Kant 1965: XI 34); in der Nachfolge Kants bestimmt Dilthey das „Erlebnis“ zum transzendentalen Ausgangspunkt seiner Hermeneutik der Geschichte (Dilthey 1970: 93); im Anschluss an Kant sieht Baumgartner in der „Kontinuität“ die maßgebende „regulative Idee“, die durch die ebenso transzendental gefasste Erzählung konstituiert wird (Baumgartner 1973: 11 f., 261); in der analytischen Geschichtsphilosophie von Danto und der folgenden Narratologie wird die Geschichte von der Erzählung konstituiert (Danto 1974: 232 ff.). 


\section{Philosophie der Geschichte}

Die zzeite Ebene betrifft die Theorie oder Philosophie der Geschichte bzw. die theoretische Geschichtsphilosophie. Hier geht es um das Verhältnis von Handlungstheorie und Methodologie. Thematisiert werden nicht bestimmte Handlungen in der Vergangenheit, sondern diejenigen Handlungskonzepte, die von Theoretikern und Philosophen vorausgesetzt werden. In einer Rekonstruktion der Methodologien historischer Erkenntnis soll deutlich werden, dass auch auf dieser Ebene ein bestimmtes Vorverständnis von Handlung zugrunde liegt. Mimesis I und II heißt in diesem Fall:

In den Geschichtstheorien, Methodologien und Geschichtsphilosophien operiert man nicht nur mit Alltagsvorstellungen über Handlungen, sondern mit verallgemeinerten und reflektierten Handlungsbegriffen, Handlungstypen und Handlungstheorien. Diese inhaltlichen Vorannahmen entscheiden letztlich über die Methode der Forschung und Darstellung. Der materiale Handlungsbegriff bestimmt, welche Art der Erklärung in der Geschichtsschreibung zum Zuge kommt. Damit präfiguriert das theoretische Vorverständnis menschlichen Handelns die entsprechende Methodologie. Dies lässt sich am Beispiel von drei idealtypisch gewählten Methoden demonstrieren: der kausalen, funktionalen und intentionalen Erklärung.

Kausale Erklärungen beziehen sich auf die äußeren Umstände des Handelns. Sie wurden bedeutsam seit der Epoche der Aufklärung, in der man bestrebt war, den Prozess der Geschichte aus ihren „Ursachen“ heraus zu erklären. Die genaue Betrachtung kann zeigen, dass damit keine Kausalkette historischer Ereignisse gemeint war, sondern die Analyse der Bedingungen kultureller Veränderungen. Diese Tradition wurde bis Marx und bis zu den sozialwissenschaftlich ausgerichteten Forschungen und Geschichtstheorien des 20. Jahrhunderts fortgesetzt. In dem Maße, in dem technologische, ökonomische, soziologische und politische Faktoren eine Rolle spielen, gewinnt die Kausalerklärung an Bedeutung. So ist ihre Verwendung ein Gradmesser dafür, welche Rolle die technische Zivilisation der Moderne in der Geschichtsphilosophie spielt.

Davon ist die funktionale Erklärung zu unterscheiden, welche die Veränderung eines Zustands oder die Entwicklung eines sozialen Systems begründet. Zu fragen ist, welche Faktoren welche Funktionen ausgeübt haben, um einen späteren Zustand zu hemmen oder zu fördern. Auch dieser Erklärungstyp ist mit der Tradition des Geschichtsdenkens zu vereinbaren. So stellt die Teleologie der klassischen Geschichtsphilosophie letztlich eine funktionale Erklärung dar. Das gilt auch für Marx, der die Geschichtsteleologie kritisiert und damit auch unabhängig von ihr funktionale Argumente benutzt. Es versteht sich von selbst, dass die moderne Systemtheorie und Evolutionstheorie mit funktionalen Erklärungen operieren. Aber dieser Erklärungstyp ist auch mit der Erzähltheorie vereinbar, weil ja vom ,Ende` her erzählt wird, wie ein bestimmter historischer Zustand entstanden 
ist und welche Ereignisse zum Erreichen dieses Zustandes beigetragen haben. In diesem Sinn kann man von einer narrativen Argumentation oder eben von einer narrativen Erklärung sprechen.

In unserem Zusammenhang geht es vor allem um intentionale Erklärungen, die sich auf die Intentionen von Handlungen beziehen. Dadurch entsteht eine gewisse Nähe zur Hermeneutik des Historismus, der sich darum bemühte, historische Ereignisse zu „verstehen“, indem die Handlungsmotive der Akteure bestimmt wurden. Bekanntlich hat sich ein scheinbarer Gegensatz zwischen ,Erklären' und ,Verstehen' gebildet, der in der analytischen Handlungstheorie des 20. Jahrhunderts dadurch aufgelöst werden konnte, dass die Motive des Handelnden als Ursachen eines historischen Ereignisses interpretiert wurden. Auf diese Weise ließen sich kausale und intentionale Erklärungen miteinander vermitteln. Die Erzähltheorie von Danto setzt die Intentionen von Handlungen und damit die intentionale Erklärung wieder außer Kraft, weil sie die Absichten der Akteure angesichts der späteren Wirkungen für unwesentlich hält. Ricœur begründet seine Hermeneutik und Phänomenologie mit einer Rehabilitierung der Handlungen einschließlich ihrer Intentionen.

Ich sehe hier noch eine andere Art der Vermittlung, wenn nämlich die intentionale Erklärung nicht nur auf individuelle, sondern auch auf kollektive Handlungen und Intentionen zielt. Droysen und Dilthey zeigen, dass sich die „Ausdrücke“ von Intentionen auch auf soziale und kulturelle Institutionen beziehen können: bei Droysen auf „sittliche Mächte“ oder „Gemeinsamkeiten“ (Droysen 1977: $291 \mathrm{ff}$., $313 \mathrm{ff}$., insb. $336 \mathrm{f}$.), bei Dilthey auf „Wirkungszusammenhänge“ oder „Kultursysteme“ (Dilthey 1970: 191 ff.). Dabei sind die Zuordnungen noch klärungs- und differenzierungsbedürftig, da ökonomische, politische oder kulturelle Zusammenhänge nicht systematisch unterschieden werden. Hier wird ein Unterschied eingeebnet, der für Marx besonders wichtig war, die Unterscheidung nämlich zwischen „naturwüchsigen“ und „bewussten“ Prozessen (Marx/Engels 1965: 52 f.). In jedem Fall schließt die funktionale Erklärung das Kollektive mit ein.

Im Historismus des 19. Jahrhunderts war die Wendung zu den Handlungen und Intentionen mit zwei kritischen Abgrenzungen verbunden: auf der einen Seite gegen die Geschichtsteleologie der Aufklärung bis Hegel, die in meiner Lesart funktionale Erklärungen anbot; auf der anderen Seite gegen die sozialwissenschaftliche Geschichtstheorien seit der Aufklärung bis Marx, in denen auch die kausale Erklärung wichtig war. Wenn nun der Historismus die intentionale Erklärung rehabilitiert, dann hängt das mit der Distanzierung von der modernen Zivilisation zusammen.

Doch weil dieser Zusammenhang nicht zwingend ist, liegt die Möglichkeit einer Vermittlung im kollektiven Handeln, das soziale Prozesse und sozialwissenschaftliche Erklärungen impliziert. Auf diese Weise lassen sich auch Intention und Kontingenz miteinander vereinbaren. Die methodischen Schwierigkeiten des Historikers, Intentionen von Handelnden sicher zu bestimmen, sprechen 
nicht gegen die Annahme von Intentionen. Auch wenn Intentionen nicht eindeutig nachweisbar sind, behält die intendierte Handlung ihre heuristische Funktion. Das gilt auch für den Fall, dass Intentionen häufig ,überdeterminiert“ sind, indem sich mehrere Intentionen kreuzen oder einander verdecken. Es gilt schließlich auch für das Phänomen der „unbeabsichtigten Nebenfolge“, weil die Intention die Bezugsgröße und der Vergleichsmaßstab bleibt, an dem Abweichungen gemessen werden. Die Behauptung des Unbeabsichtigten setzt die Vorstellung einer beabsichtigten Wirkung voraus.

Die intentionale Erklärung ist also mit der Grunderfahrung historischer Kontingenz vereinbar, wie Droysen und Ricœur zeigen (Droysen 1977: 164; Ricœur 1986: 12 f., 16 f.). Droysen hatte noch die Teleologie von Hegel bemüht, um diese Erfahrung zu formulieren. Aber andere Autoren wie Marx und Ricœur zeigen, dass Kontingenz auch ohne Teleologie gedacht werden kann. Vor allem aber schließt Kontingenz die Spielräume für individuelles und kollektives Handeln nicht aus. Im Gegenteil, dadurch eröffnen sich gerade neue Möglichkeiten für intentionales Handeln und für intentionale Erklärungen. Eine solche Erweiterung des Handlungsfeldes ist im Hinblick auf eine Ethik der Geschichte geboten, wenn die Verantwortung für menschliches Handeln zur Diskussion steht.

\section{Pragmatik}

Die dritte Ebene betrifft das pragmatische Verbältnis von Geschichtsschreibung und gesellschaftlicher Praxis.

Einerseits ist die Deutung von Geschichte bis hin zur Darstellung und Erzählung selbst eine gesellschaftliche Praxis. Die Erzeugung von Deutungssinn wird von praktischen Interessen geleitet und damit von einem vorgängigen Handlungssinn diktiert. So wie man die gesellschaftliche Praxis als einen „Diskurs“ bezeichnet hat, so kann man unter dem Diskurs auch eine Art Handlung verstehen, d. h. eine Sprach- oder Erzählhandlung. Diese Umkehr ist nicht beliebig, weil damit behauptet wird, dass historische Erzählungen eine praktische Funktion ausüben. Im günstigen Fall dient sie der Orientierung in historischen Situationen im Hinblick auf Zukunftsfragen. Im schlechteren Fall dient sie der Legitimation von Herrschaft, wenn etwa im Namen des „Fortschritts“ Opfer verlangt werden.

Andererseits wirkt die Historiographie auf die gesellschaftliche Praxis zurück, wo sie selbst eine praktische Funktion ausübt. Deutungsmuster des Historischen dienen den Akteuren zur Orientierung, indem sie ihren Handlungen, insbesondere ihren Motiven und Intentionen, einen bestimmten Sinn verleihen. Politiker deuten ihr eigenes Handeln ständig als „historisch“, sprechen von „historischen Augenblicken“ oder handeln im Hinblick auf spätere Anerkennung. Mit Hilfe derartiger Deutungsmuster sind individuelle und kollektive Subjekte imstande, sich über ihre Handlungen und Erwartungen zu verständigen. Dies geschieht vor allem bei Hand- 
lungen mit langfristiger Perspektive. Die Menschen handeln etwa, um den „Fortschritt" voranzubringen, um beispielsweise die Klimakatastrophe abzuwenden oder um überhaupt für die zukünftigen Generationen Vorsorge zu treffen (Rohbeck 2013: 37 ff.). In solchen Fällen beeinflusst der Deutungssinn den Handlungssinn.

Dieser zweite Aspekt der Pragmatik der Geschichtserzählung kommt insbesondere in der Theorie des Gedächtnisses zum Ausdruck (Ricœur 2004: 95 ff.). Während die historische Erzählung rückwärts in die Vergangenheit gewandt ist, steht das Gedächtnis für die Orientierung in der Gegenwart und für die Projektion in die Zukunft. Außerdem repräsentiert es die gesellschaftliche Dimension des Geschichtsbewusstseins, indem es, im Unterschied zur Erzählung, als kollektives Gedächtnis begriffen wird. Dabei geht es um aktuelle Fragen der Erinnerungskultur einer Gesellschaft oder einer Nation, um Fragen des Gedenkens an frühere Ereignisse und damit auch des Ignorierens bestimmter Ereignisse. Gefragt wird nach dem moralischen und politischen Umgang mit der Schuld früherer Generationen, bis hin zu Fragen der Bestrafung und Amnestie. In diesen praktischen Kontexten spielt die Historiographie eine besondere Rolle.

Nach Ricœur sollen sich Gedächtnis und Geschichtsschreibung wechselseitig ergänzen (223 ff.). Einerseits korrigiert das kollektive Gedächtnis die Rückwärtsgewandtheit und den Wissenschaftspositivismus der Historiker; es bewegt den Historiker auch dazu, selbst als politische Person Stellung zu nehmen und sich praktisch zu verhalten. Andererseits bedarf das kollektive Gedächtnis der Korrektur durch die Historiker, welche den Wahrheitsanspruch zu prüfen haben, indem sie Fakten klären und Deutungen anbieten. An dieser Stelle schlägt sich die Geschichtserzählung in der Lebenspraxis nieder. Der narrative Deutungssinn verbindet sich mit dem praktischen Handlungssinn.

\section{Aktualisierung in praktischer Absicht}

Aus guten Gründen kann man die Geschichtsphilosophie als Ausdruck der Erfahrung von der „Unverfügbarkeit“ der Geschichte interpretieren. Folgt man dieser These, lässt sich die Teleologie der Geschichte als Kompensation dieser Einsicht deuten: entweder als pure Ideologie oder als psychische Angstbewältigung in einer pathologischen Moderne (Koselleck 1979: 158, 260 ff.; Lübbe 1977: 25; Kittsteiner 1980: 163 f.). Doch stelle ich mir eine andere Frage. Anstelle ideologiekritischer und sozialpsychologischer Verdächtigungen frage ich, ob die Geschichtsphilosophie nicht auch Modelle bereithält, die zu einer vernünftigen Bewältigung historischer Kontingenz beitragen können (Ricœur: 1986: 11 ff.; Rohbeck 2013: 104 ff.). Lassen sich in den Denkfiguren der Geschichtsphilosophie Ansätze einer rationalen Verarbeitung entdecken? So setze ich an die Stelle von Ohnmachtsphantasien die Aufgabe, die Spielräume theoretischer Reflexion, praktischer Eingriffe und historischer Verantwortung auszuloten. 
Indem ich mich dieser praktischen Frage zuwende, verlasse ich die Ebene der Geschichtserzählung und wende mich wieder der dritten Stufe der Mimesis von Ricœur zu. Damit kehre ich von Fragen der narrativen und erklärenden Deutung zum Handlungssinn der Lebenspraxis zurück. Da ich keine reine Methodologie betreibe, bewege ich mich von der formalen zur materialen Geschichtsphilosophie. Ich frage, welche Orientierungsleistung von den soeben behandelten Konzeptionen für die Lösung künftiger Aufgaben zu erwarten ist.

Dazu sind noch einmal die in den Geschichtstheorien und Geschichtsphilosophien aufgetretenen Handlungsmodelle sowie ihre Beziehung zur historischen Kontingenz in Erinnerung zu rufen. So wie wir zwischen mehreren Modellen menschlichen Handelns unterschieden haben, so gibt es auch mehrere Arten der Kontingenz, die dazu im Verhältnis stehen. Die genannten Handlungsmodelle mit ihren entsprechenden Methoden enthalten offenbar unterschiedliche Arten von Kontingenz und bilden in ihren Beziehungen zueinander unterschiedliche Kontingenzverhältnisse.

Das Modell des kollektiven Handelns spielte zu Beginn der neuzeitlichen Geschichtsphilosophie insofern eine Rolle, als im Zuge der politischen Ökonomie die Eigendynamik ökonomischer Systeme erkannt wurde. Diese Systeme stellten das Gegenteil von Kontingenz dar, sie wurden nach dem Muster von Naturgesetzen betrachtet, so wie auch die soziale Evolution geradezu wie ein naturaler Prozess betrachtet wurde. Vom Standpunkt des beginnenden Liberalismus war diese „natürliche Ordnung" sogar erwünscht. Erst bei Marx zeigten sich Kontingenzen sowohl innerhalb des kapitalistischen Systems, dessen „Krisen“ zunehmend zum Vorschein kamen, als auch zwischen den sozialen Teilsystemen Ökonomie, Politik und Kultur. Schließlich diagnostizierte Marx eine Kontingenz des Kapitalismus gegenüber den einzelnen Menschen, welche den „naturwüchsigen“ Markt als Entfremdung und damit als Einschränkung ihrer Autonomie erfahren. Marx war es denn auch, der gegen diese unterschiedlichen Kontingenzen das Modell des politischen Handelns und damit die kollektive Intention einer sozialen Klasse aufbot.

Im Anschluss an diese Entwicklung stellt sich eine Reihe von Fragen: Die Macht der sozialen Systeme schränkt die Möglichkeiten des intentionalen Handelns ein, das demgegenüber als kontingent erfahren wird. Auf der anderen Seite eröffnet die Kontingenz des kollektiven Handelns in den sozialen Systemen gerade auch die Möglichkeit des intentionalen Handelns. Daraus folgt, dass mit der Perspektive praktischer Eingriffe auf die Kontingenzen der sozialen Systeme zu achten ist; sie können auch als Chance für praktische, verändernde Eingriffe verstanden werden. Die entscheidende Frage der Gegenwart besteht darin, welche Spielräume intentionalen Handelns die sozialen Systeme überhaupt zulassen. Wenn die Evolution dieser Systeme als historischer Determinismus erscheint, kommt es darauf an, die Aufmerksamkeit auf Kontingenzen zu richten, welche ein eingreifendes Handeln ermöglichen. Wenn heute der sich beschleunigende Prozess der Modernisierung als eine Steigerung von Kontingenz beschrieben 
wird, gewinnt die Frage nach Kontingenzbewältigung eine aktuelle Bedeutung nicht nur im Sinne eines angepassten Verhaltens, sondern gerade auch als kritisch-verändernder Eingriff.

Gegenüber diesen Erfahrungen mit der modernen Zivilisation kann man den Historismus wie eine romantische Reaktion interpretieren. Denn er verlagerte den Akzent auf Politik, Religion und Kultur. Damit sollten die intentionalen Handlungen der Individuen wieder stärker zur Geltung kommen. Nun waren es die Handlungen der Individuen, die als Quelle von Kontingenz galten. Der Hinweis auf die Tatsache, dass letztlich unberechenbare Menschen die Geschichte „machen“, diente zur Charakterisierung des spezifischen Gegenstandes Geschichte. Dieses Argument setzte sich in der analytischen Geschichtsphilosophie gegen die naturwissenschaftlich orientierte Kausalerklärung durch. Der Einwand war daher berechtigt, der Historiker müsse auch mit irrationalen, also kontingenten Handlungsmotiven rechnen.

Setzt man das intentionale Handeln, das geradezu den Prototyp von Kontingenz in der Geschichtswissenschaft ausmacht, ins Verhältnis zum kollektiven Handeln, gilt das oben Gesagte in umgekehrter Richtung. Gegen die Übermacht der Systeme ist, sofern sich durch systemische Kontingenz Lücken auftun, an der relativen Macht der Intentionen festzuhalten. Außerdem verbindet sich damit die normative Dimension menschlichen Handelns. Ist doch die Forderung unhintergehbar, die Individuen für ihr in bestimmten Grenzen intentionales Handeln verantwortlich zu machen. Aus der Perspektive der Handelnden heißt dies: Sie sind für die späteren Folgen ihres Handelns verantwortlich. Auch wenn die Macht der sozialen Systeme erdrückend zu sein scheint, bleibt nichts anderes übrig, als auf das intentionale Handeln der Menschen zu hoffen, um verändernde Eingriffe offen zu lassen.

\section{Literatur}

Ankersmit, Frank (2009) „Danto's Philosophy of History in Retrospective“. Journal of the Philosophy of History 3.2: 109-45.

Aron, Raymond (1948) Introduction à la philosophie de l'histoire: Essai sur les limites de l'objectivité historique. Paris: Gallimard.

Baumgartner, Hans Michael (1973) Kontinuität und Geschichte: Zur Kritik und Metakritik der historischen Vernunft. Frankfurt a. M.: Suhrkamp.

Bubner, Rüdiger (1984) Geschichtsprozesse und Handlungsnormen: Untersuchungen zur praktischen Philosophie. Frankfurt a. M.: Suhrkamp.

Danto, Arthur C. (1974) Analytische Philosophie der Geschichte. Frankfurt a. M.: Suhrkamp.

Dilthey, Wilhelm (1970) Der Aufbau der geschichtlichen Welt in den Geisteswissenschaften. Hg. Manfred Riedel. Frankfurt a. M.: Suhrkamp. 
Droysen, Johann Gustav (1977) Historik. Textausgabe von Peter Leyh. StuttgartBad Cannstatt: Frommann-Holzboog.

Dux, Günter (1989) Die Zeit in der Geschichte: Ihre Entwicklungslogik vom Mythos zur Weltzeit. Frankfurt a. M.: Suhrkamp.

Haussmann, Thomas (1991) Zur Theorie und Pragmatik der Geschichtswissenschaft. Frankfurt a. M.: Suhrkamp.

Hegel, Georg Wilhelm Friedrich (1969) Werke in 20 Bänden. Hgg. Eva Moldenhauer und Karl Markus Michel. Frankfurt a. M.: Suhrkamp.

Hempel, Carl G. (1959) „The Function of General Laws in History“. Theories of History. Hg. Patrick Gardiner. New York: Free Press. 344-56.

Kant, Immanuel (1965) Werke in 12 Bänden. Hg. Wilhelm Weischedel. Frankfurt a. M.: Suhrkamp.

Kittsteiner, Heinz Dieter (1980) Naturabsicht und Unsichtbare Hand. Frankfurt a. M.: Ullstein.

Kocka, Jürgen (1989) Geschichte und Aufklärung. Göttingen: Vandenhoeck \& Ruprecht.

Koselleck, Reinhart (1979) Vergangene Zukunft. Zur Semantik geschichtlicher Zeiten. Frankfurt a. M.: Suhrkamp.

Lorenz, Chris (1997) Konstruktion der Vergangenheit: Eine Einfübrung in die Geschichtstheorie. Köln: Böhlau.

Lübbe, Hermann (1977) Geschichtsbegriff und Geschichtsinteresse: Analytik und Pragmatik der Historie. Basel: Schwabe.

Marx, Karl, und Friedrich Engels (1965) Werke: Briefe, Januar 1868 - Mitte Juli 1870. Band 32. Berlin: Dietz.

Müller-Funk, Wolfgang (2002) Die Kultur und ibre Narrative: Eine Einfübrung. Wien/New York: Springer.

Ricœur, Paul (1986) „Contingence et Rationalité dans le Récit“. Studien zur neueren französischen Phänomenologie: Phänomenologische Forschungen 18. Hg. Ernst Wolfgang Orth. Freiburg/München: Alber. 11-29.

- (1988-91) Zeit und Erzäblung. 3 Bde. München: Fink.

- (1997) „Gedächtnis - Vergessen - Geschichte“. Historische Sinnbildung: Problemstellungen, Zeitkonzepte, Wahrnehmungshorizonte, Darstellungsstrategien. Hgg. Klaus E. Müller und Jörn Rüsen. Reinbek bei Hamburg: Rowohlt. 43354.

- (1998) Das Rätsel der Vergangenheit: Erinnern - Vergessen - Verzeiben. Göttingen: Wallstein-Verlag.

- (2004) Gedächtnis, Geschichte, Vergessen. München: Fink.

Riedel, Manfred (1978) Verstehen oder Erklären? Zur Theorie und Geschichte der hermeneutischen Wissenschaften. Stuttgart: Klett-Cotta.

Rohbeck, Johannes (2000) Technik - Kultur - Geschichte. Eine Rehabilitierung der Geschichtsphilosophie. Frankfurt a. M.: Suhrkamp. 
- (2010): Aufklärung und Geschichte: Eine praktische Geschichtsphilosophie der Zukunft. Berlin: Akademie.

- (2013): Zukunft der Geschichte: Geschichtsphilosophie und Zukunftsethik. Berlin: Akademie.

Schiffer, Werner (1980) Theorien der Geschichtsschreibung und ibre erzäbltheoretische Relevanz: Danto, Habermas, Baumgartner, Droysen. Stuttgart: Metzler.

Schnädelbach, Herbert (1987) Vernunft und Geschichte: Vorträge und Abhandlungen. Frankfurt a. M.: Suhrkamp.

- (2003) „Kulturelle Evolution“. Geschichtsphilosophie und Kulturkritik: Historische und systematische Studien. Hgg. Johannes Rohbeck und Herta Nagl-Docekal. Darmstadt: WBG. 329-51.

Stückrath, Jörn, und Jürg Zbinden (1997) Hgg. Metageschichte: Hayden White und Paul Ricour. Baden-Baden: Nomos Verlagsgesellschaft.

Wright, Georg Henrik von (1974) Erklären und Verstehen. Königstein: Athenäeum. White, Hayden (1986) Auch Klio dichtet. Stuttgart: Klett-Cotta.

- (1990) Die Bedeutung der Form: Erzäblstrukturen in der Geschichtsscbreibung. Frankfurt a. M.: Fischer.

- (1991) Metahistory: Die historische Einbildungskraft im 19. Jahrbundert in Europa. Frankfurt a. M.: Fischer. 
